\title{
Tutoría por pares de distinto año académico en la enseñanza de habilidades quirúrgicas básicas en estudiantes de pregrado de medicina
}

\author{
Roberto González L. ${ }^{1,2}$, Héctor Molina Z. ${ }^{2}$, María García-Huidobro D. ${ }^{2,3}$, \\ Patricio Stevens M. ${ }^{2,3}$, Andrés Jadue T. ${ }^{1}$, Alejandra Riquelme U. ${ }^{2}$, \\ Javier Torres M. ${ }^{2}$, Als. Felipe Alarcón O. ${ }^{5}$ Sebastián Barra M. ${ }^{5}$, \\ Claudio Toloza A. ${ }^{5}$, Matías Ávalos T. ${ }^{5}$ y Eduardo Fasce H. ${ }^{4}$
}

Peer tutoring in the teaching of basic surgical skills in medical undergraduate students

\begin{abstract}
Aim: Our objective is to compare the effectiveness of tutoring taught by 6 th versus 14 th semester peer students in the acquisition of basic procedural skills in medical students. Materials and Method: Quasi experimental study, before and after. The intervention initially consists of a training workshop for suture monitors (SM) for two students of 6 th and two students of 14 th semester of medicine. Subsequently, a suture workshop taught by SM to 23 fourth-year medical students was implemented. Continuous and discontinuous suture was evaluated using the instrument: "Objective structured evaluation of technical skills" (OSATS) and satisfaction through a survey validated by experts. The results of pre and post intervention and student satisfaction are described and compared, according to the semester attended by the SM. Informed consent was applied. Excel ${ }^{\circledR}$ and SPSS2 $4^{\circledR}$ were used for statistical analysis by Mann Whitney test. It was considered significant $\mathrm{p}<0.05$. Results: Both groups progressed significantly on the OSATS scale, group tutored by $6^{\circ}$ semester SM: 18.1 vs $28,1(\mathrm{p}<0.001)$ and group tutored by $14^{\circ}$ semester SM: 15.5 vs 27,8 and $(\mathrm{p}<0.001)$. The final scores did not they present significant differences. The intervention was well evaluated with 66 out of 68 points, without significant differences according to the semester coursed of the SM. Conclusion: Our experience shows that it is possible to use peer tutoring by trained tutors to teach surgical skills, regardless of whether the teaching is given by pairs of lower or higher courses, achieving significant progress and equivalent procedural performance, in addition to high satisfaction among students.
\end{abstract}

Key words: surgery; medical education; undergraduate; simulation training; peer.

\section{Resumen}

Objetivo: Nuestro objetivo es comparar la efectividad de la tutoría impartida por pares de $6^{\circ}$ versus $14^{\circ}$ semestre en la adquisición de habilidades procedimentales básicas en estudiantes de medicina. Materiales y Método: Estudio cuasi-experimental, antes y después. La intervención consta inicialmente de un curso de formación de monitores de sutura (MS) para dos estudiantes de $6^{\circ}$ y dos de $14^{\circ}$ semestre de medicina. Posteriormente se implementó un taller de sutura tutorizado por MS a 23 estudiantes de $8^{\circ}$ semestre de medicina. Se evaluó sutura continua y discontinua mediante el instrumento: "The Objective Structured Assessment Of Technical Skills” (OSATS) y satisfacción mediante encuesta tipo Likert validada por expertos. Se describen y comparan los resultados pre y postintervención y satisfacción de los estudiantes, de acuerdo al semestre cursado del MS. Se aplicó consentimiento informado. Se utilizó SPSS24 ${ }^{\circledR}$ para análisis estadístico mediante prueba de Mann Whitney. Se consideró significativo $p<0,05$. Resultados: Ambos grupos progresaron significativamente en la escala OSATS, grupo tutorizado por MS de $6^{\circ}$ semestre: 18,1 vs 28,1 $(\mathrm{p}<0,001)$ y grupo tutorizado por MS de $14^{\circ}$ semestre: 15,5 vs $27,8(\mathrm{p}<0,001)$. Los puntajes finales no presentan diferencias significativas. La intervención fue evaluada con 66 de 68 puntos, sin diferencias
'Centro Cardiovascular, Hospital Clínico Regional de Concepción Dr. Guillermo Grant Benavente.

${ }^{2}$ Departamento de Cirugía, Facultad de Medicina,

Universidad de Concepción. ${ }^{3}$ Servicio de Cirugía, Hospital Clínico Regional de Concepción Dr. Guillermo Grant Benavente.

${ }^{4}$ Departamento de Educación Médica, Facultad de Medicina Universidad de Concepción

${ }^{5}$ Estudiante Medicina, Facultad Medicina, Universidad de Concepción. Concepción, Chile.

Recibido el 5 de febrero de 2019 y aceptado para publicación el 4 de abril de 2019

Correspondencia a: Dr. Roberto González L. rgonzalezlagos@udec.cl 
significativas según el semestre cursado del MS. Conclusión: Nuestra experiencia muestra que es posible utilizar la tutoría por pares entrenados para la enseñanza de habilidades quirúrgicas, independientemente si la tutoría es impartida por pares de cursos inferiores o superiores, logrando un progreso significativo y un desempeño procedimental equivalente, además de una alta satisfacción entre los estudiantes.

Palabras clave: cirugía; educación médica; pregrado; entrenamiento de simulación; pares.

\section{Introducción}

El desarrollo de habilidades quirúrgicas básicas en estudiantes de pregrado de medicina es un requisito dentro de los programas curriculares, sin embargo, los egresados refieren una falta de competencia y habilidad al término de su formación ${ }^{1-6}$. Los métodos de enseñanza de habilidades quirúrgicas presentan enormes variaciones entre instituciones educativas, así, muchos estudiantes adquieren estas destrezas de forma independiente, aludiendo al interés propio en el área quirúrgica, cursos externos, residentes becarios de cirugía o a cirujanos docentes ${ }^{7}$.

Las variaciones en los métodos de enseñanza de estas competencias provocan que las primeras experiencias en suturas sean realizadas en pacientes en ausencia de una base teórica sólida y de un entrenamiento retroalimentado en cuanto a la técnica. Esto implica una posible transgresión de la seguridad del paciente y genera un ambiente de aprendizaje estresante. Esto, unido a las dificultades en el proceso de aprendizaje en pregrado se han asociado a una disminución de la confianza del estudiante y una propensión a evitar realizar procedimientos como suturas a lo largo de la carrera, y a largo plazo, ha mostrado afectar negativamente las habilidades psicomotoras, incrementando los errores y la imprecisión $^{8,9}$.

El uso de simuladores ha ganado popularidad entre las escuelas de medicina debido a ofrecer un entorno seguro y regulado donde los estudiantes pueden aprender y errar, resultando con un mejor entrenamiento previo al contacto con los pacientes $^{10-12}$.

La tutoría por pares es una metodología que consiste en un aprendizaje cooperativo entre estudiantes, donde un grupo de ellos toma un rol como educadores frente a los otros. Este método ha demostrado beneficios que se justifican a partir de la premisa de que los pares tienen experiencias similares, comprendiendo de mejor manera el desarrollo del aprendizaje ${ }^{13}$. No obstante, existe una falta de consenso en relación a su efectividad en la adquisición de habilidades a corto y largo plazo y el desarrollo de habilidades clínicas ${ }^{14}$.

Nuestra experiencia mostró que la enseñanza por parte de docentes cirujanos y pares entrenados puede lograr un progreso equivalente y significativo en la adquisición de habilidades procedimentales básicas ${ }^{15,16}$. Cabe señalar que la tutoría por pares tradicionalmente ha consistido en estudiantes de cursos inferiores bajo la tutoría de estudiantes de cursos superiores ${ }^{17}$. Existe poca evidencia sobre la equivalencia de la tutoría por pares de cursos inferiores frente a la tutoría por pares de cursos cercanos a finalizar su formación de pregrado.

Nuestro objetivo es comparar la efectividad de la tutoría impartida por pares de $6^{\circ}$ versus $14^{\circ}$ semestre en la adquisición de habilidades procedimentales básicas en estudiantes de medicina.

\section{Materiales y Método}

Se realizó un estudio cuasi-experimental, antes y después. Se comparó la adquisición de habilidades procedimentales mediante un taller de sutura basado en modelo biológico, en dos grupos de estudiantes de $8^{\circ}$ semestre de medicina mediante la tutoría por pares. Un grupo fue entrenado por dos monitores de sutura (MS) que no habían comenzado sus actividades clínicas $\left(6^{\circ}\right.$ semestre), mientras el segundo estuvo bajo la tutoría de dos MS del último semestre de la carrera de medicina $\left(14^{\circ}\right.$ semestre $)$ que cursaron el internado de cirugía correspondiente. La implementación y estudio de una intervención tutorizada para la enseñanza de habilidades quirúrgicas para estudiantes de medicina fue aprobada por el comité de ética de la Facultad de Medicina de la Universidad de Concepción.

\section{Curso de formación de monitores de sutura}

La intervención constó inicialmente de un curso que incluyó a dos estudiantes de $6^{\circ}$ semestre y dos de $14^{\circ}$ semestre de medicina, según muestreo por conveniencia, durante el periodo mayo-junio del año 
$2017^{18}$. Se firmó consentimiento informado. Dicho curso tuvo una duración de 28 h y se dividió en tres módulos:

\section{Módulo de clases teóricas integrales}

De ocho horas, impartida por cirujanos plásticos docentes, a modo de entregar conocimientos amplios acerca de la técnica de sutura incluyendo historia, comportamiento físico y biológico, recomendaciones actuales, uso de instrumental quirúrgico, técnica aséptica, anestesia local, cuidados postoperatorios y manejo de complicaciones.

Al finalizar, los estudiantes rindieron una evaluación de conocimientos teóricos con una nota de aprobación mínima de 6,0 (escala de 1,0 a 7,0).

\section{Módulo de simulación en modelo biológico}

Se utilizó como modelo biológico una pata de cerdo (cruda, refrigerada y no congelada) sobre una tabla de madera enchapada fijada a ella con ligas. Se puso a disposición suturas de seda 2-0 e instrumental quirúrgico básico (porta aguja, pinza quirúrgica y tijera recta roma aguda). Se realizó una incisión de aproximadamente 5 centímetros. Se dispuso de material de limpieza y depósito de material cortopunzante.

El módulo consistió en diez horas de práctica bajo la tutoría de un cirujano cardiotorácico quien entregó herramientas prácticas de enseñanza en pequeños grupos.

\section{Módulo de práctica en pabellón quirúrgico}

Los MS asistieron a diez horas de pabellón quirúrgico en cirugía cardiotorácica, previo consentimiento informado de los pacientes y en estricta supervisión de cirujanos subespecialistas. El objetivo fue la práctica in vivo de sutura de distintos planos en herida quirúrgica de safenectomía y esternotomía, exposición al ambiente quirúrgico de una cirugía compleja con trabajo multidisciplinario y la posibilidad de realizar seguimiento de la herida quirúrgica en postoperatorio y control ambulatorio.

Todos los participantes lograron un progreso significativo en sus habilidades procedimentales y obtuvieron una calificación que les permitió aprobar el curso y ser reconocidos como MS de la Facultad de Medicina de la Universidad de Concepción.

\section{Implementación de taller de sutura}

Se implementó un taller tutorizado por MS para 23 estudiantes de $8^{\circ}$ semestre de la carrera de Medicina que cursaban la asignatura de cirugía en la Universidad de Concepción durante el segundo semestre de 2017. Todos los participantes firmaron consentimiento informado. Los estudiantes fueron divididos aleatoriamente en grupos bajo la tutoría de MS de $6^{\circ}$ y $14^{\circ}$ semestre ( 11 y 12 estudiantes, respectivamente). Cada grupo de estudiantes tutorizados estuvo compuesto por 5 o 6 estudiantes.

\section{Módulo teórico}

Previo al módulo práctico se puso a disposición de los participantes material audiovisual consistente en tres videos de elaboración propia (disponibles en la plataforma ARCO de nuestra Universidad) con una duración no superior a 10 min. Ellos exponen: conceptos básicos, materiales de sutura, anestesia local, instrumental quirúrgico básico y una demostración tutorial de infiltración anestésica y técnica de sutura.

\section{Módulo práctico}

Se utilizó el mismo modelo de simulación descrito para la formación de MS. El taller se realizó en las dependencias del Departamento de Cirugía de la Facultad de Medicina y se desarrolló siempre bajo la supervisión del autor principal (RGL).

El taller contempló 16 h y se dividió en cuatro sesiones, una sesión semanal de cuatro horas, donde se tutorizó acerca de las tareas: preparación de campo quirúrgico, técnica aséptica, infiltración anestésica, uso del instrumental quirúrgico, nudos con porta aguja y nudos manuales.

Al iniciar el taller, los estudiantes fueron expuestos a un ejercicio de sutura continua y discontinua en el modelo biológico (herida simulada de piel de muslo y piel cabelluda, respectivamente), en un plazo de cinco minutos. El mismo ejercicio se ha llevado a cabo al finalizar el taller y en ambos casos los estudiantes utilizaron teléfonos móviles para registrar en video la técnica de sutura con el objetivo de facilitar la evaluación.

\section{Evaluación}

El desempeño de los estudiantes fue evaluado empleando el instrumento: "The Objective Structured Assessment Of Technical Skills" (OSATS)"19. Se utilizó la sección "escala global OSATS" que consta de siete ítems en formato de escala Likert (con puntajes posibles de 1 al 5, en caso de evaluarse el aspecto de forma ineficiente o si posee un dominio óptimo, respectivamente).

Los ítems evaluados corresponden a: "manejo de tejidos", "tiempo y motricidad", "manipulación de instrumentos", "uso apropiado del instrumental quirúrgico", "planificación y ritmo", "conocimiento del procedimiento específico" y "utilización de ayudantes." Se adaptó el instrumento eliminando el 


\section{ARTÍCULO ORIGINAL}

ítem "utilización de ayudantes" en concordancia con los objetivos de la investigación, resultando en una escala de 6 puntos mínimos y 30 puntos máximos.

Los videos registrados previo y posterior a la intervención fueron recopilados y sus nombres de archivo fueron aleatorizados y codificados mediante números para ser finalmente visualizados por cirujanos especialistas ajenos al taller de sutura, ciegos a la temporalidad de los videos y al grupo de MS al que pertenecía el estudiante.

Se evaluó la satisfacción de los estudiantes mediante una encuesta tipo Likert de 17 ítems validada por expertos del Departamento de Educación Médica de la Facultad de Medicina de la Universidad de Concepción $^{20}$. El instrumento abarca los ámbitos: generalidades del taller, desempeño de tutores, materiales y satisfacción de los estudiantes.

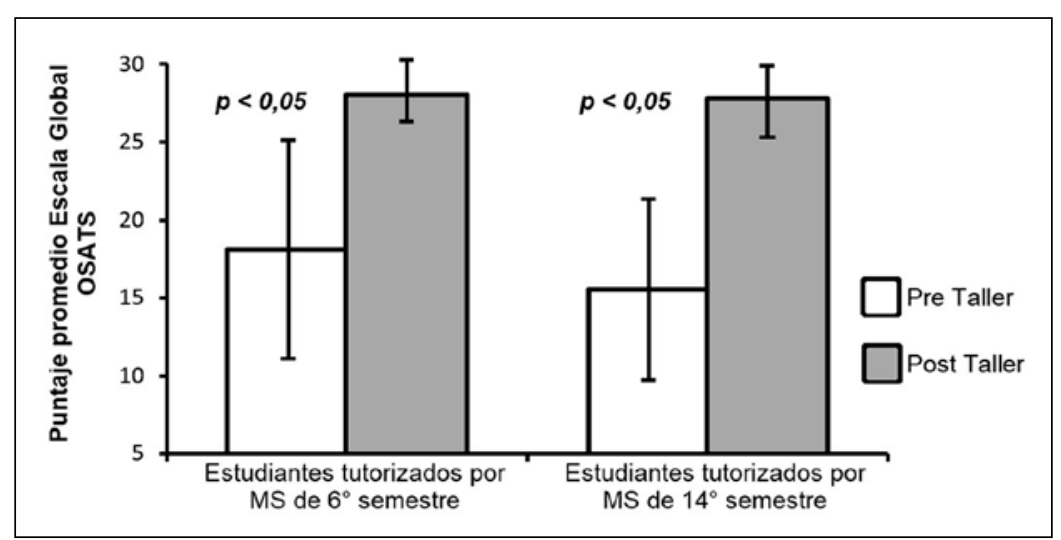

Figura 1. Resultados escala global OSATS, puntajes promedio pre y postaller a estudiantes de $8^{\circ}$ semestre, según la tutoría impartida por monitores de $6^{\circ}$ y $14^{\circ}$ semestre. $\mathrm{MS}=$ Monitor de sutura.
Se describen los resultados pre y postintervención y la satisfacción de los estudiantes, comparando los grupos bajo la tutoría de estudiantes pertenecientes a un semestre curricular inferior $\left(6^{\circ}\right.$ semestre $)$ versus el grupo bajo la tutoría de estudiantes del último semestre curricular $\left(14^{\circ}\right.$ semestre). Se utilizó SPSS $24^{\circledR}$ para análisis de datos y se usó la prueba de Mann Whitney para variables no paramétricas. Se consideró significativo $\mathrm{p}<0,05$.

\section{Resultados}

Los 23 participantes cumplieron con un 100\% de asistencia. En promedio, el universo de los participantes muestra un progreso significativo en el puntaje total de la escala global OSATS posterior a la intervención (16,8 a 27,9 puntos, $\mathrm{p}<0,05)$.

Ambos grupos, estudiantes tutorizados por MS de $6^{\circ}$ y $14^{\circ}$ semestre muestran un progreso significativo en los puntajes totales de la escala global OSATS (Figura 1), sin diferencias significativas en los puntajes finales de la misma escala $(28,1$ versus 27,8 puntos, respectivamente), con un valor $\mathrm{p}=0,48$, lo que se observa en la Tabla 1.

Los ítems de la escala global OSATS que muestran un menor rendimiento previo al taller corresponden a "Tiempo y motricidad" (2,3 versus 2,8 puntos en grupos bajo tutoría de MS de $6^{\circ}$ semestre versus $14^{\circ}$ semestre) y "Manipulación de instrumentos" ( 2,8 versus 2,3 puntos en grupos bajo tutoría de MS de $6^{\circ}$ semestre versus $14^{\circ}$ semestre). Los ítems mencionados, por lo demás, obtuvieron la mayor progresión absoluta al finalizar la intervención en ambos grupos comparados (Figura 2).

Tabla 1. Resultados escala global OSATS posterior al taller de suturas realizado a estudiantes de $8^{\circ}$ semestre de Medicina, comparación entre la tutoría por pares de $6^{\circ}$ semestre versus $14^{\circ}$ semestre

\begin{tabular}{|lccc|}
\hline & $\begin{array}{c}\text { Estudiantes tutorizados } \\
\text { por MS de } \mathbf{6}^{\circ} \text { semestre }\end{array}$ & $\begin{array}{c}\text { Estudiantes tutorizados } \\
\text { por MS de 14 }\end{array}$ semestre & Valor p \\
\hline Manejo de tejidos & $4,8 \pm 0,2$ & $4,6 \pm 0,9$ & 0,12 \\
\hline Tiempo y motricidad & $4,5 \pm 0,4$ & $4,7 \pm 0,8$ & 0,36 \\
\hline Manipulación de instrumentos & $4,56 \pm 0,4$ & $4,7 \pm 0,6$ & 0,40 \\
\hline Uso apropiado del instrumental & $4,7 \pm 0,3$ & $4,9 \pm 0,1$ & 0,30 \\
\hline Planificación y ritmo & $4,8 \pm 0,3$ & $4,9 \pm 0,3$ & 0,50 \\
\hline Conocimiento del procedimiento específico & $4,8 \pm 0,4$ & $4,8 \pm 0,6$ & 0,41 \\
\hline Total & $28,1 \pm 1,7$ & $27,8 \pm 2,5$ & 0,48 \\
\hline
\end{tabular}

MS = Monitor de sutura 
ARTÍCULO ORIGINAL

Al comparar la percepción de los estudiantes frente a la metodología implementada mediante la encuesta de satisfacción (68 puntos máximos) se obtiene un puntaje promedio de 65,5 en el grupo entrenado por MS de $6^{\circ}$ semestre versus 66,6 en el grupo entrenado por MS de $14^{\circ}$ semestre, sin diferencias significativas con un valor $\mathrm{p}=0,548$ (Figura 3 ).

\section{Discusión}

La enseñanza y adquisición de habilidades quirúrgicas en estudiantes de medicina de pregrado representa un reto para las facultades de medicina. Circunstancias como la necesidad de enseñanza personalizada, necesidad de cirujanos docentes, resguardo de la seguridad de los pacientes y escasez de situaciones de la vida real han dificultado el desarrollo de este tipo de aprendizaje?

La simulación en la enseñanza de procedimientos quirúrgicos ofrece ventajas como la entrega de conocimientos teórico-prácticos sin comprometer los asuntos éticos inherentes de la práctica en pacientes, mejora la comunicación entre el estudiante-tutor, facilita la transición del estudiante al quehacer clínico y favorece el aprendizaje en un entorno menos estresante que el pabellón quirúrgico o la sala de urgencias $^{21,22}$. Los beneficios de la simulación están sustentados, entre otros motivos, debido a que la retención y consolidación de la habilidad procedimental es favorecida por un modelo de múltiples y breves instancias de práctica y una variedad en la secuencia de las tareas comprometidas ${ }^{23}$. Estos elementos se buscaron replicar en el taller expuesto, organizando una sesión por semana, de corta duración e intercalando las distintas tareas.

Por otro lado, la dificultad de contar con un gran número de cirujanos docentes necesarios para la enseñanza personalizada ha fomentado la utilización de pares para la enseñanza de habilidades quirúrgicas básicas como suturas simples ${ }^{15,16}$.

La enseñanza por pares se ha asociado a una menor distancia entre tutor-estudiante, grupos de menor tamaño y un ambiente de baja formalidad ${ }^{24}$. Entre sus beneficios, Moust y Schmidt ${ }^{13}$, sostienen que los tutores-pares son capaces de entender mejor las dificultades de sus estudiantes debido a que poseen experiencias de aprendizaje similares, además, los estudiantes tienden a externalizar sus pensamientos más que con docentes formales, permitiendo corregir errores inadvertidos en el aprendizaje con mayor frecuencia.

Estudios han demostrado que la formación previa del tutor es un factor crucial en el éxito de esta me-

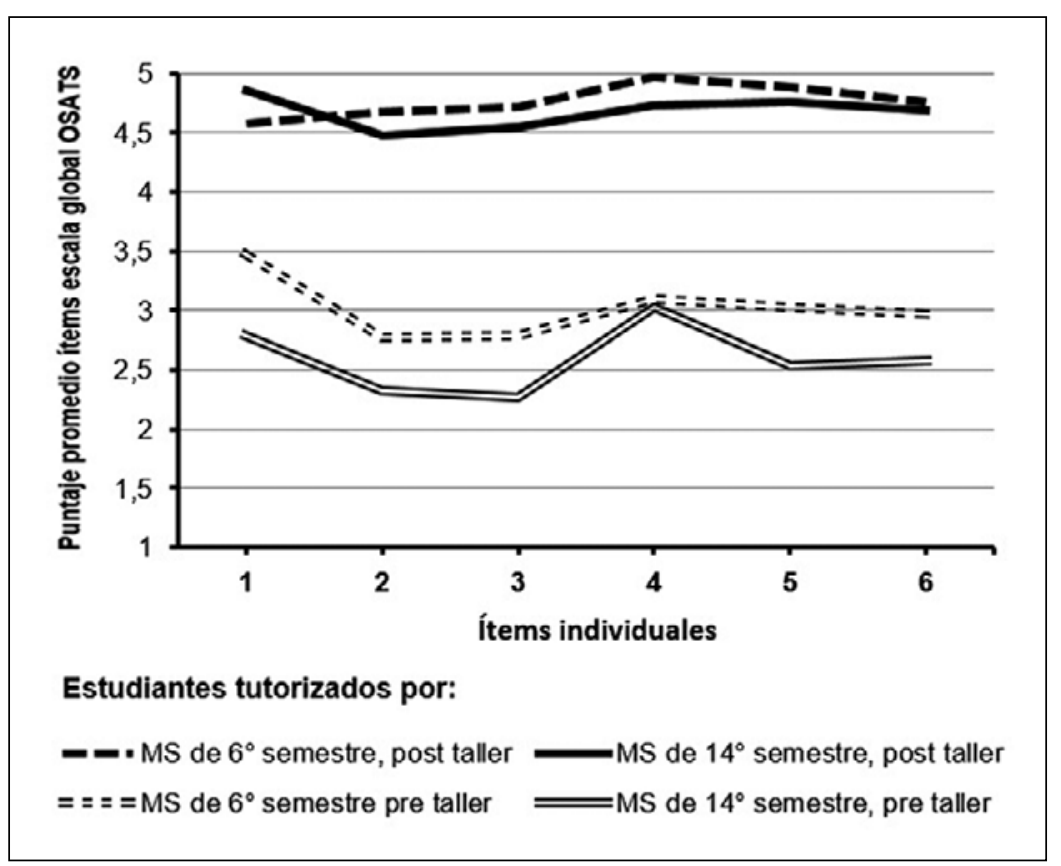

Figura 2. Resultados escala global OSATS por ítems, puntajes promedio pre y post taller, según tutoría impartida por monitores de $6^{\circ}$ semestre versus monitores de $14^{\circ}$ semestre. MS: Monitor de sutura. Leyenda: (1) Manejo de tejidos, (2) Tiempo y motricidad, (3) Manipulación de instrumentos, (4) Uso apropiado del instrumental, (5) Planificación y ritmo, (6) Conocimiento del procedimiento específico.

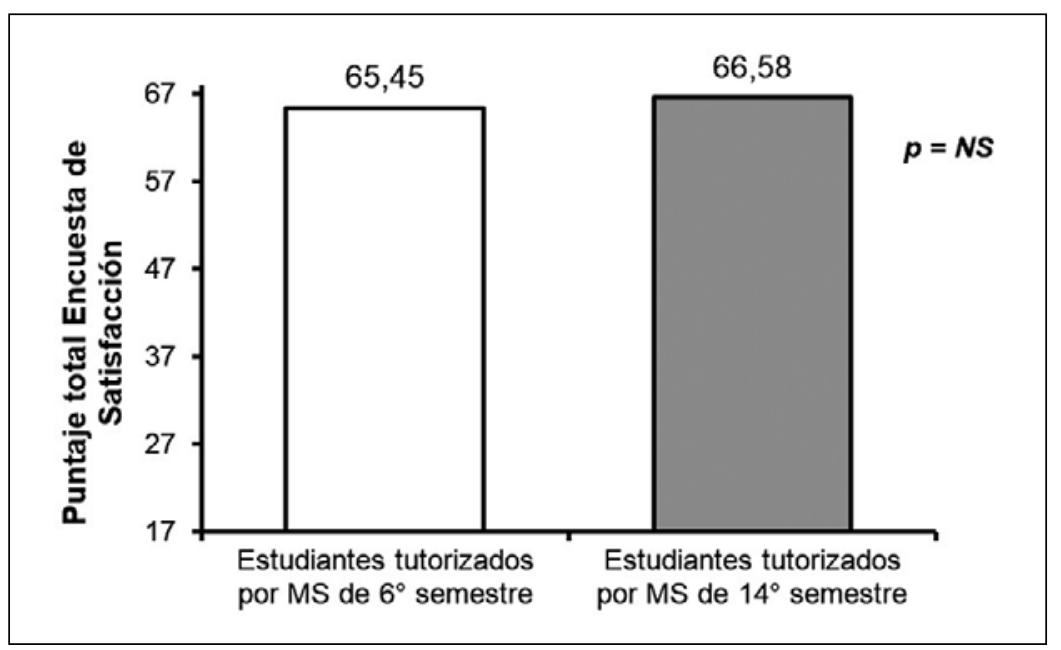

Figura 3. Puntaje total promedio de encuesta de satisfacción a estudiantes de $8^{\circ}$ semestre, según tutoría impartida por monitores de $6^{\circ}$ y $14^{\circ}$ semestre. MS $=$ Monitor de sutura; NS = No significativo.

todología $a^{25}$ y han descrito que la retroalimentación objetiva sumado a una evaluación certera son los factores con mayor influencia en el aprendizaje de habilidades motoras ${ }^{26,27}$, destacando el rol de un par entrenado como un agente activo que identifique y solucione errores en el aprendizaje. 
Por otra parte, se ha comparado la eficacia de un taller de sutura comparando el uso de un software de aprendizaje versus la instrucción por un instructor experto enfocado en la retroalimentación, dando como resultado un mayor desempeño de este último grupo en la enseñanza de técnica de sutura y nudo con manos $^{10}$. De manera similar, estudios han mostrado una superioridad de la retroalimentación verbal de un experto versus un software que evalúa y corrige el procedimiento a partir de la eficiencia del movimiento ${ }^{26}$.

Pocas investigaciones han analizado los efectos de utilizar pares de cursos inferiores versus pares de cursos superiores. En nuestra metodología, la diferencia fundamental entre los monitores consiste en que los estudiantes de $6^{\circ}$ semestre no habían iniciado sus prácticas clínicas, mientras que los estudiantes de $14^{\circ}$ semestre habían cumplido con su formación en cirugía incluyendo el internado correspondiente, asumiendo entonces una importante brecha en conocimiento teórico-práctico previa formación de los MS.

Es posible identificar al menos dos beneficios prácticos de la utilización de pares de cursos inferiores: los pares entrenados son capaces de desempeñarse como tutores por más tiempo a lo largo de la carrera y en ellos es previsible un desarrollo de habilidades docentes y un reconocimiento de las falencias más frecuentes en los estudiantes debido a la mayor práctica.

Una limitación de este estudio es el número acotado de participantes debido a ser desarrollado en el contexto de la tesis del autor principal. Actualmente, nuestro equipo trabaja en la implementación del taller a la totalidad de los estudiantes del cuarto año durante la asignatura impartida por el Departamento de Cirugía, lo que será materia de futuras investigaciones. Por otro lado, el instrumento OSATS utilizado para evaluar el desempeño procedimental no incluye en su estructura una valorización que permita distinguir un puntaje reprobatorio de uno aceptable u óptimo, sin embargo, un progreso significativo en el puntaje que se acerca al máximo de la escala en ambos grupos se ha considerado un resultado exitoso para la intervención. Por último, la metodología cuasi-experimental obliga valorizar los resultados con cautela, esta metodología se utilizó por lo cuestionable que sería generar un grupo de control y no incluir a la totalidad de los estudiantes en una intervención que asumimos beneficiosa.

En conclusión, nuestra experiencia muestra que es posible utilizar la tutoría por pares entrenados para la enseñanza de habilidades quirúrgicas, independientemente si la tutoría es impartida por pares de cursos inferiores o superiores, logrando un progreso significativo y un desempeño procedimental equivalente, además de una alta satisfacción entre los estudiantes.

\section{Responsabilidades éticas}

Protección de personas y animales. Los autores declaran que para esta investigación no se han realizado experimentos en seres humanos ni en animales.

Confidencialidad de los datos. Los autores declaran que en este artículo no aparecen datos de pacientes.

Conflictos de interés: Este trabajo se enmarca dentro de la Tesis titulada "Competencias procedimentales quirúrgicas básicas en estudiantes de medicina, enseñanza práctica por docentes y por pares", para optar al grado de Magíster en Educación Médica para Ciencias de la Salud, a cargo del investigador principal.

\section{Bibliografía}

1. Cumming A, Ross M. The Tuning Project for Medicine-learning outcomes for undergraduate medical education in Europe. Med Teach 2007;29:636-41.

2. Davis C, Toll E, Bates A, Cole M, Smith F. Surgical and procedural skills training at medical school - a national review. Int J Surg. 2014;12:877-82.

3. Dehmer J, Amos K, Farrell T, Meyer A, New-ton W, Meyers M. Competence and confidence with basic procedural skills: the experience and opinions of fourth-year medical students at a single institution. Acad Med. 2013;88:682-7.

4. Villegas F, Polaco A, González J, García A, Madrid M. Competencias médicoquirúrgicas. Autopercepción en médicos recién egresados de la licenciatura. Cir Ciruj. 2007;75:43-7.

5. Coberly L, Goldenhar L. Ready or not, here they come: acting interns' experience and perceived competency performing basic medical procedures. J Gen Intern Med. 2007;22:491-4.
6. Fincher R, Lewis L. Learning, experience, and self-assessment of competence of third-year medical students in performing bedside procedures. Acad Med. 1994;69: 291-5.

7. Lyons-Warren A, Kirby J, Larsen D. Student views on the role of self-regulated learning in a surgery clerkship. J Surg Res. 2016;206:273-9.

8. Arora S, Sevdalis N, Aggarwal R, Sirimanna P, Darzi A, Kneebone R. Stress impairs psychomotor performance 
in novice laparoscopic surgeons. Surg Endosc. 2010;24:2588-93.

9. Clanton J, Gardner A, Cheung M, Mellert L, Evancho-Chapman M, George R. The relationship between confidence and competence in the development of surgical skills. J Surg Educ. 2014;71:40512.

10. Xeroulis G, Park J, Moulton C, Reznick R, Leblanc V, Dubrowski A. Teaching suturing and knot-tying skills to medical students: a randomized controlled study comparing computer-based video instruction and (concurrent and summary) expert feedback. Surgery 2007;141:442-9.

11. Lentz G, Mandel L, Goff B. A six-year study of surgical teaching and skills evaluation for obstetric/gynecologic residents in porcine and inanimate surgical models. Am J Obstet Gynecol. 2005;193:2056-61.

12. Alvarado J, Henríquez J, Castillo R, Sosa J, León F, Varas J, et al. Programa pionero de simulación en sutura para estudiantes de medicina de pregrado. Rev Chil Cir. 2015;67:480-5.

13. Moust J, Schmidt H. Effects of staff and student tutors on student achievement Higher Education 1994;28:471-2.

14. Secomb J. A systematic review of peer teaching and learning in clinical education. J Clin Nurs. 2008;17:703-16.

15. González R. Competencias procedimentales quirúrgicas básicas en estudiantes de medicina, enseñanza práctica por docentes y por pares [master's thesis]. Concepción: Universidad de Concepción; 2018.124p. Disponible en: http://repositorio.udec.cl/ handle/11594/2991

16. González R, Molina H, García-Huidobro M, Stevens P, Jadue A, Riquelme A, et al. Basic suture techniques for medicine students: comparative results according to training by surgeons versus peers. Cir Cir. 2019; [In press].

17. Bennett S, Morris S, Mirza S. Medical Students Teaching Medical Students Surgical Skills: The Benefits of Peer-Assisted Learning. J Surg Ed. 2018;75:1471-4.

18. González R, Molina H, García-Huidobro M, Stevens P, Jadue A, Riquelme A, et al. Implementación de taller de monitores de sutura en alumnos de pregrado de medicina. Rev Chil Cir. 2018;71:122-8.

19. Martin J, Regehr G, Reznick R, MacRae $\mathrm{H}$, Mur-Naghan J, Hutchison C, et al. Objective structured assessment of technical skill (OSATS) for surgical residents. Br J Surg. 1997;84:273-8.

20. González R, Molina H, García-Huidobro M, Stevens P, Jadue A, Riquelme A, et al. Percepción y grado de satisfacción de estudiantes de medicina sobre implementación de taller de suturas: enseñanza práctica por docentes y por pares en la asignatura de cirugía. Rev Educ Cienc Salud 2018;15:87-91.

21. Manning P, Mishall L, Weidmann D,
Flax H, Lan S, Erlich M, et al. Early and prolonged opportunities to practice suturing increases medical student confort with suturing during clerckships: suturing during cadaver dissection. Anat Sci Educ. 2018;605-12.

22. LaPorta J, McClore T, Tieman M. Results of a surgical simulation course designed to improve surgery clerkship performance. $\mathrm{J}$ Surg Educ. 2018;75:656-63.

23. Nicholls D, Sweet L, Muller A, Hyett J. Teaching psychomotor skills in the twenty-first century: revisiting and reviewing instructional approaches through the lens of contemporary literature. Med Teach 2016;38:1056-63.

24. Ten Cate O, Durning S. Dimensions and psychology of peer teaching in medical education. Med Teach 2007;29: 546-52.

25. Shiozawa T, Hirt B, Lammerding-Koeppel $M$. The influence of tutor training for peer tutors in the dissection course on the learning behavior of students. Ann Anat. 2016;208:212-6.

26. Porte M, Xeroulis G, Reznick R, Dubrowski A. Verbal feedback from an expert is more effective than self-accessed feedback about motion efficiency in learning new surgical skills. Am J Surg. 2007;193:105-10.

27. Vanyolos E, Furka I, Miko I, Viszlai A, Nementh N, Peto K. How Does practice improve the skills of medical students during consecutive training courses?. Acta Cir Bras. 2017;32:491-502. 